\title{
Gravitational perturbations of the Kerr black hole due to arbitrary sources
}

\author{
Claudia Moreno [and Darío Núñez † \\ Center for Gravitational Physics 83 Geometry \\ Penn State University, University Park, PA 16802 \\ and \\ ${ }^{1}$ Departamento de Física, \\ Centro de Investigación y de Estudios Avanzados del I. P. N., \\ A. P. 14-740, 07000 México, D.F., MEXICO \\ ${ }^{2}$ Instituto de Ciencias Nucleares - UNAM, \\ Apdo. 70-543, Ciudad Universitaria, 04510 Mexico, D.F.
}

\begin{abstract}
We describe the Kerr black hole in the ingoing and outgoing Kerr-Schild horizon penetrating coordinates. Starting from the null vector naturally defined in these coordinates, we construct the null tetrad for each case, as well as the corresponding geometrical quantities allowing us to explicitly derive the field equations for the $\Psi_{0}{ }^{(1)}$ and $\Psi_{4}{ }^{(1)}$ perturbed scalar projections of the Weyl tensor, including arbitrary source terms. This perturbative description, including arbitrary sources, described in horizon penetrating coordinates is desirable in several lines of research on black holes, and contributes to the implementation of a formalism aimed to study the evolution of the space time in the region where two black holes are close.
\end{abstract}

PACS:95.35.+d, 95.35.G

\footnotetext{
${ }^{1}$ E-mail: yaya@fis.cinvestav.mx

${ }^{2}$ E-mail: nunez@nuclecu.unam.mx
} 


\section{Introduction}

A particle falling unto a black hole is an efficient mechanism for generation of gravitational waves. This problem is not only of great importance to gravitatonal wave astrophysics, but it also has important application of numerical general relativity.

The fact that several gravitational observatories (LIGO, VIRGO, GEO600, and later LISA)will start to be working soon is one of the most exciting news lately received by the general relativistic community. Those observatories will allow a much deeper insight on the phenomena near the black holes such as the above mentioned particle falling unto it, and later, will allow us to see beyond the recombination era, plus all the not yet expected ones.

These technological achievements, together with a strong development in the computational techniques, definitely boost the great theoretical knowledge acquired in the last century. In particular, the theoretical study on gravitational perturbations goes back to Einstein and has produce excellent works such as the one by Chadrasekhar [1] or the one by Frolov [2], for just to mention a couple of them. Also, there is the work by Teukolsky [3] who, using the Newman Penrose formalism for null tetrads (see [1, 2]), was able to obtain an equation for the ingoing and outgoing radiative parts of the perturbed Weyl tensor, namely $\Psi_{0}{ }^{(1)}$ and $\Psi_{4}{ }^{(1)}$, respectively, which were separable in the frequency domain. The radial part of those equation were useful in obtaining information about the asymptotic behavior of the gravitational perturbations in the vacuum case.

The complexity of the equations prevented further advances until the development of numerical techniques for studding the evolution of the equations. There was large effort in understanding the collision of black holes evolving directly the Einstein equations [1, 5], as well as those studies comprising semi analytical results where they used the perturbation equations derived by Teukolsky with two important changes: First, with a coordinate system which avoids the coordinate singularity, and second, they worked in the time domain, to be able to study the evolution.

Campanelli et al. [6] presented a work in this last direction and obtained the evolution of the $\Psi_{4}{ }^{(1)}$ Weyl tensor for the case without sources. In the present work, we continue within such line of research, obtaining the equations which allow the study by numerical evolution of the $\Psi_{0}{ }^{(1)}$ and $\Psi_{4}{ }^{(1)}$ perturbed Weyl scalars in the case with arbitrary sources generating the perturbation. Furthermore, families of initial data with appealing features such as describing regularly multiple moving spinning black holes [7], use the Kerr Schild horizon penetrating coordinates.

We stress the fact that in the present work we explicitly derive the field equations for the $\Psi_{0}{ }^{(1)}$ and $\Psi_{4}{ }^{(1)}$ perturbed scalar projections of the Weyl tensor, including arbitrary source terms. Even though

there is the coordinate transformation from the Boyer Lindquist to the Kerr Schild ones, the change of tetrad involves rotations and dilatation of the null tetrad which prevent to treat the Teukolsky equation as a tensor operator, and it is necessary to derive the equations from their general definitions, as is done in the present work.

The work is composed as follows. In the next section we derive the Kerr metric in ingoing and outgoing Kerr Schild coordinates, and present the field equations for $\Psi_{0}{ }^{(1)}$ and $\Psi_{4}{ }^{(1)}$, deriving the master field equation. Next, we take the naturally defined null vector and construct the null tetrad, the differential operators, the spinor coefficients and the Weyl scalars and, describing the main steps in the deduction, we finally derive explicitly the expression for the perturbation equations for arbitrary sources valid in a Kerr Schild description, in the ingoing as well as in the outgoing formulation. We test our result in simple examples and conclude mentioning several case where will be of interest apply the obtained equations.

\section{Kerr Schild line element and master perturbation equation}

The rotating black hole is usually described by the line element in Boyer Lindquist coordinates [8]:

$$
d s^{2}=\frac{\Delta}{\Sigma}\left(d t-a \sin ^{2} \theta d \varphi\right)^{2}-\frac{\sin ^{2} \theta}{\Sigma}\left[\left(r^{2}+a^{2}\right) d \varphi-a d t\right]^{2}-\Sigma\left(\frac{d r^{2}}{\Delta}+d \theta^{2}\right),
$$


with $\Delta=r^{2}-2 M r+a^{2}$, and $\Sigma=r^{2}+a^{2} \cos ^{2} \theta$.

However, as mentioned above, this description has a divergence at the external horizon: $r_{+}=M(1+$ $\left.\sqrt{1+\left(\frac{a}{M}\right)^{2}}\right)$, which prevent that the evolution analysis could be performed in the vicinity of the external horizon. Moreover, it has been established that the gravitational wave emission of several phenomena such as the matter accretion or the inspariling of an object unto the black hole, occurs precisely within this region [9. Detailed studies show that there is a sweep upward in frequency, during the last 15 minutes before the collision and final coalescence, that is, precisely in the region close to the external horizon for which the Boyer Lindquist coordinates are not well suited.

Thus it is imperative to be able to perform the description of the phenomena within a coordinate system which does not have those coordinate problems. Whit in this spirit the Kerr Shild description not only avoids the problem of coordinate singularities at the horizon but is the most natural description to be matched with the null tetrad formulation. In this way, following [1], we define the coordinates $t$ and $\varphi$ in terms of the outgoing and ingoing ones, $u_{\substack{\text { in } \\ \text { out }}}$ :

$$
d u u_{\text {in }}^{\text {out }}=d t \pm \frac{r^{2}+a^{2}}{\Delta} d r, \quad d \varphi \underset{\substack{\text { in } \\ \text { out }}}{\Delta}=d \varphi \pm \frac{a}{\Delta} d r,
$$

and, defining in each case a new temporal coordinate

$$
d t_{\text {out }}^{\text {in }}=d u \underset{\text { out }}{\text { out }} \mp d r
$$

We obtain that the Kerr space time is described as:

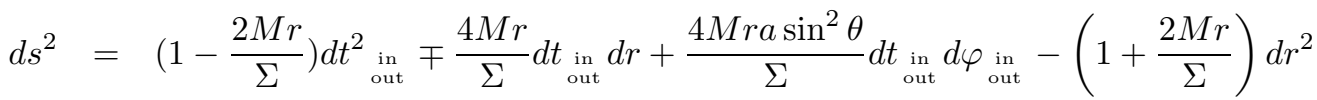

$$
\begin{aligned}
& \pm 2\left(1+\frac{2 M r}{\Sigma}\right) a \sin ^{2} \theta d r d \varphi_{\substack{\text { out } \\
\text { out }}}-\Sigma d \theta^{2}-\left(r^{2}+a^{2}+\frac{2 M r a^{2} \sin ^{2} \theta}{\Sigma}\right) \sin ^{2} \theta d \varphi_{\substack{\text { out } \\
\text { out }}},
\end{aligned}
$$

which is the known Kerr Schild description. Noticing that this last expression for the Kerr line element, Eq. (4) can be rewritten as:

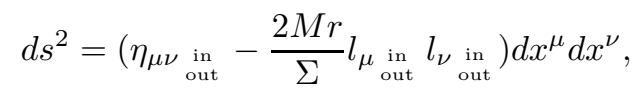

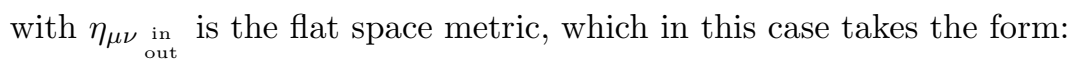

$$
\eta_{\mu \nu} \underset{\text { out }}{\text { in }} d x^{\mu} d x^{\nu}=d t^{2} \underset{\text { out }}{\text { in }}-d r^{2}-\Sigma d \theta^{2}-\left(r^{2}+a^{2}\right) \sin ^{2} \theta d \varphi^{2} \underset{\text { out }}{\text { in }} \pm 2 a \sin ^{2} \theta d r d \varphi \underset{\text { out }}{\text { on }},
$$

and $l_{\mu \text { in }}$ is a null vector, null as much for the flat metric as for the complete one, and is given by:

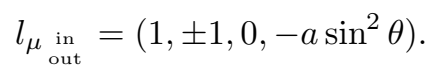

We want to stress the fact that, as mentioned above, the line element in the Kerr Schild coordinates, Eq.(车), not only has no geometric divergence all the way from infinity to the interior of the black hole, but also defines naturally one of the null vectors.

Thus, we use this null vector, defined by Eq.(7), to construct the null tetrad, the differential operators, the spinor coefficients and the Weyl scalars, which are needed to study the gravitational perturbations within the Newman Penrose formulation. The null tetrad $l, n, m$ and $m^{*}$ is defined by the following identities:

$$
l \cdot n=1 \quad \text { and } \quad m \cdot m^{*}=-1,
$$

and from the relation of the tetrad with the metric coefficients:

$$
g_{\mu \nu}=2\left[l_{(\mu} n_{\nu)}-m_{(\mu} m^{*}{ }_{\nu)}\right] .
$$


Before presenting explicitly the ingoing and outgoing cases, we derive the field equations for the perturbed Weyl components, valid for any algebraically special type D space time.

Perturbing the Bianchi identities in the NP formulation [1, 2], taking into account the expressions derived in the above section, coupled equations for the perturbed Weyl scalars $\Psi_{0}{ }^{(1)}$ and $\Psi_{1}{ }^{(1)}$ and $\Psi_{3}{ }^{(1)}$ $\Psi_{4}{ }^{(1)}$, are obtained. Using the generalized commutation relations between the spinor operators given in the appendix A, Eq.(37), as well as the perturbed Ricci identities obtained form [1, 2] and the expressions derived in the above section, the following separate equations for the perturbed Weyl scalars $\Psi_{0}{ }^{(1)}$ and $\Psi_{4}{ }^{(1)}$ are obtain:

$$
\begin{aligned}
& \left\{\left(\mathbf{D}-4 \rho-\rho^{*}-3 \varepsilon+\varepsilon^{*}\right)(\boldsymbol{\Delta}+\mu-4 \gamma)-(\delta-2 \beta-4 \tau)\left(\delta^{*}+4 \beta^{*}-3 \pi\right)-3 \Psi_{2}\right\} \Psi_{0}{ }^{(1)} \\
& =8 \pi_{2} \mathcal{T}^{\mu \nu} T_{\mu \nu}{ }^{(1)}, \\
& \left\{\left(\boldsymbol{\Delta}+4 \mu+\mu^{*}+3 \gamma-\gamma^{*}\right)(\mathbf{D}-\rho+4 \varepsilon)-\left(\delta^{*}-2 \beta^{*}+7 \pi-\tau^{*}\right)(\delta+4 \beta-\tau)-3 \Psi_{2}\right\} \Psi_{4}{ }^{(1)} \\
& =8 \pi_{-2} \mathcal{T}^{\mu \nu} T_{\mu \nu}{ }^{(1)},
\end{aligned}
$$

where ${ }_{s} \mathcal{T}^{\mu \nu}$ are given by:

$$
\begin{aligned}
& { }_{2} \mathcal{T}^{\mu \nu}=-(\delta-2 \beta-4 \tau)\left(\delta-\pi^{*}\right) l^{\mu} l^{\nu}-\left(\mathbf{D}-4 \rho-\rho^{*}-3 \varepsilon+\varepsilon^{*}\right)\left(\mathbf{D}-\rho^{*}-2 \varepsilon+2 \varepsilon^{*}\right) m^{\mu} m^{\nu} \\
& +\left[(\delta-2 \beta-4 \tau)\left(\mathbf{D}-2 \rho^{*}-2 \varepsilon\right)+\left(\mathbf{D}-4 \rho-\rho^{*}-3 \varepsilon+\varepsilon^{*}\right)\left(\delta-2 \beta+2 \pi^{*}\right)\right] l^{(\mu} m^{\nu)}, \\
& -2 \mathcal{T}^{\mu \nu}= \\
& -\left(\delta^{*}-2 \beta^{*}+7 \pi-\tau^{*}\right)\left(\delta^{*}+2 \pi-\tau^{*}\right) n^{\mu} n^{\nu}-\left(\boldsymbol{\Delta}+4 \mu+\mu^{*}+3 \gamma-\gamma^{*}\right)\left(\boldsymbol{\Delta}+\mu^{*}+2 \gamma-2 \gamma^{*}\right) m^{* \mu} m^{* \nu} \\
& +\left[\left(\delta^{*}-2 \beta^{*}+7 \pi-\tau^{*}\right)\left(\boldsymbol{\Delta}+2 \mu^{*}+2 \gamma\right)+\left(\boldsymbol{\Delta}+4 \mu+\mu^{*}+3 \gamma-\gamma^{*}\right)\left(\delta^{*}-2 \beta^{*}+2 \pi-2 \tau^{*}\right)\right] n^{(\mu} m^{* \nu)},
\end{aligned}
$$

with $T_{\mu \nu}{ }^{(1)}$ is the source of the perturbation. These equations were first derived by Teukolsky in 1972, [3] and, as long as he was interested in the asymptotic behavior, the Eddington Finkelstein coordinates which he used, were well suited for the analysis.

The studies are performed on the $\Psi_{0}{ }^{(1)}$ and $\Psi_{4}{ }^{(1)}$ perturbed Weyl scalars as long as those are the most significant components in the gravitational radiation [3].

Acting on $\rho^{-4} \Psi_{4}{ }^{(1)}$ and using the commutation relations between the operators, Eq.(37), and the action of the operators on the spinor coefficients, Eq.(39) into the appendix A, the perturbation equations for $\Psi_{0}{ }^{(1)}$ and $\rho^{-4} \Psi_{4}{ }^{(1)}$, Eqs.(11), can be written as a single master equation in terms of the parameter $s$ :

$$
\begin{aligned}
& \left\{\boldsymbol{\Delta} \mathbf{D}-\delta^{*} \delta+\left(\mu^{*}-(2 s+1) \gamma-\gamma^{*}\right) \mathbf{D}-((2 s+1) \rho+2 s \varepsilon) \boldsymbol{\Delta}-\left(2(s+1) \beta^{*}-(2 s+1) \pi-\tau^{*}\right) \delta\right. \\
& +(2 s \beta+(2 s+1) \tau) \delta^{*}+s(2 s+1)\left[2(\rho+\varepsilon) \gamma-2 \beta \pi-2 \pi \tau+2 \beta^{*} \tau-\Psi_{2}\right]- \\
& \left.2 s\left[(\boldsymbol{\Delta} \varepsilon)-\left(\delta^{*} \beta\right)+\varepsilon\left(\mu^{*}-\gamma^{*}\right)+\beta \tau^{*}\right]+4 s(s+1) \beta \beta^{*}\right\}_{s} \psi=8 \pi \rho^{(s-2)}{ }_{s} \mathcal{T}^{\mu \nu} T_{\mu \nu}{ }^{(1)}
\end{aligned}
$$

with ${ }_{2} \psi=\Psi_{0}{ }^{(1)} \mathrm{y}_{-2} \psi=\rho^{-4} \Psi_{4}{ }^{(1)}$. Even though the $\Psi_{0}{ }^{(1)}$ and $\Psi_{4}{ }^{(1)}$ perturbed Weyl scalars are the most significant components in the gravitational radiation, it would be interesting to analyze the evolution of the rest of the perturbed Weyl scalars, $\Psi_{1}{ }^{(1)}, \Psi_{2}{ }^{(1)}$ and $\Psi_{3}{ }^{(1)}$. Some studies indicate that it is possible to obtain a single master equation for the five of the perturbed Weyl scalars [10, 11, 12]. These studies are currently under investigation and will be reported elsewhere.

\section{Null tetrad formulation and explicit derivation of the pertur- bation equations for $\Psi_{0}^{(1)}$ and $\Psi_{4}^{(1)}$ with sources}

Next, we presents the main steps in the derivation of the explicit form of the field equations for the perturbed $\Psi_{0}^{(1)}$ and $\Psi_{4}{ }^{(1)}$ Weyl components with sources. In the appendix B, we present the main expressions for the action of the operators on the spinor coefficients, needed in the derivation of the explicit equation. We are going to present both cases, the ingoing and the outgoing. 


\subsection{Ingoing case}

Starting form the naturally defined null vector, given by Eq.(7), using the equations Eqs.(8.,9), we construct the following ingoing null tetrad:

$$
\begin{aligned}
l_{\mu_{\mathrm{in}}} & =\left(1,1,0,-a \sin ^{2} \theta\right), \\
n_{\mu_{\mathrm{in}}} & =\frac{1}{2 \Sigma}\left(\Delta, r^{2}+a^{2}\left(\cos ^{2} \theta-\sin ^{2} \theta\right)+2 M r, 0,-a \Delta \sin ^{2} \theta\right), \\
m_{\mu_{\mathrm{in}}} & =\frac{1}{\sqrt{2}(r+i a \cos \theta)}\left[-i a \sin \theta,-i a \sin \theta, \Sigma, i\left(r^{2}+a^{2}\right) \sin \theta\right] .
\end{aligned}
$$

From which we obtain the covariant null vectors:

$$
\begin{aligned}
l_{\text {in }}^{\mu} & =(1,-1,0,0), \\
n^{\mu}{ }_{\text {in }} & =\frac{1}{2 \Sigma}\left(r^{2}+a^{2}+2 M r, \Delta, 0,2 a\right), \\
m^{\mu}{ }_{\text {in }} & =-\frac{1}{\sqrt{2}(r+i a \cos \theta)}(i a \sin \theta, 0,1, i \csc \theta),
\end{aligned}
$$

as well as the corresponding differential operators:

$$
\begin{aligned}
\mathbf{D}_{\mathrm{in}} & =l^{\mu}{ }_{\mathrm{in}} \partial_{\mu}=\partial_{t_{\mathrm{in}}}-\partial_{r} \\
\boldsymbol{\Delta}_{\mathrm{in}} & =n^{\mu}{ }_{\text {in }} \partial_{\mu}=\frac{1}{2 \Sigma}\left[\left(r^{2}+a^{2}+2 M r\right) \partial_{t_{\mathrm{in}}}+\Delta \partial_{r}+2 a \partial_{\varphi_{\mathrm{in}}}\right] \\
\delta_{\mathrm{in}} & =m^{\mu}{ }_{\mathrm{in}} \partial_{\mu}=-\frac{\rho_{\mathrm{in}}}{\sqrt{2}}\left(i a \sin \theta \partial_{t_{\mathrm{in}}}+\partial_{\theta}+i \csc \theta \partial_{\varphi_{\mathrm{in}}}\right)
\end{aligned}
$$

with the spinor coefficient $\rho_{\text {in }}$ defined below in Eq.(17).

The spinor coefficients are computed directly from their definition [1, 2], obtaining:

$$
\begin{aligned}
& \kappa_{\mathrm{in}}=\sigma_{\mathrm{in}}=\lambda_{\mathrm{in}}=\nu_{\mathrm{in}}=0, \\
& \rho_{\mathrm{in}}=\frac{1}{r+i a \cos \theta}, \quad 2 \varepsilon_{\mathrm{in}}=\rho_{\mathrm{in}}-\rho_{\mathrm{in}}^{*}, \quad \mu_{\mathrm{in}}=\frac{\Delta}{2 \Sigma} \rho_{\mathrm{in}}, \quad 2 \gamma_{\mathrm{in}}=\mu_{\mathrm{in}}+\mu^{*}{ }_{\mathrm{in}}-\frac{(r-M)}{\Sigma}, \\
& \pi_{\mathrm{in}}=\frac{i a \sin \theta}{\sqrt{2} \Sigma}, \quad 2 \beta_{\mathrm{in}}=-\frac{\cot \theta}{\sqrt{2}} \rho_{\mathrm{in}}+\pi_{\mathrm{in}}^{*}+\tau_{\mathrm{in}}, \quad \alpha_{\mathrm{in}}=\pi_{\mathrm{in}}-\beta_{\mathrm{in}}{ }^{*}, \quad \tau_{\mathrm{in}}=-\frac{i a \sin \theta}{\sqrt{2}} \rho_{\mathrm{in}}{ }^{2},
\end{aligned}
$$

and finally, the only non zero Weyl scalar is:

$$
\Psi_{2 \text { in }}=-M \rho_{\text {in }}^{3} \text {. }
$$

Using the explicit form of the differential operators, Eqs.(16), of the spinor coefficients and the Weyl scalar Eqs. (17, 18), for the null tetrad Eq. 15) of the Kerr metric described in Kerr Schild coordinates, Eq.(4), with the help of the expressions for the action of the operators on the spinor coefficients given in the appendix A, Eq.(39), (40), we derive the equations for the perturbations of the $\Psi_{0}^{(1)}$ and $\Psi_{4}{ }^{(1)}$ perturbed Weyl scalars due to arbitrary source terms in ingoing coordinates:

$$
\begin{aligned}
& {\left[(\Sigma+2 M r) \frac{\partial^{2}}{\partial t_{\mathrm{in}}^{2}}-4 M r \frac{\partial^{2}}{\partial r \partial t_{\mathrm{in}}}-\Delta \frac{\partial^{2}}{\partial r^{2}}-2 a \frac{\partial^{2}}{\partial r \partial \varphi_{\mathrm{in}}}-\frac{\partial^{2}}{\partial \theta^{2}}-\frac{1}{\sin ^{2} \theta} \frac{\partial^{2}}{\partial \varphi_{\mathrm{in}}^{2}}\right.} \\
& -2\left((s+1)(r-M)+2 s \Delta \varepsilon_{\mathrm{in}}\right) \frac{\partial}{\partial r}-2\left(s r+(s+1) M-s \varepsilon_{\mathrm{in}}(\Sigma+4 M r)\right) \frac{\partial}{\partial t_{\mathrm{in}}} \\
& -\left(\cot \theta+4 s r \tan \theta \varepsilon_{\mathrm{in}}\right) \frac{\partial}{\partial \theta}-2 s\left(i \frac{\cos \theta}{\sin ^{2} \theta}+4 a \varepsilon_{\mathrm{in}}\right) \frac{\partial}{\partial \varphi_{\mathrm{in}}}-s\left(1-s \cot ^{2} \theta\right)+\frac{16 a^{2}}{\Sigma}\left(1-\frac{2 M r \cos ^{2} \theta}{\Sigma}\right) \\
& \left.-4 s \varepsilon_{\mathrm{in}}\left((s+1) r+\left(r^{2}-s\left(r^{2}-a^{2} \cos ^{2} \theta\right)\right) \frac{M}{\Sigma}\right)\right]_{s} \psi=16 \pi \Sigma_{s} \mathcal{T}^{\mu \nu} T_{\mu \nu}^{(1)},
\end{aligned}
$$


where ${ }_{s} \mathcal{T}^{\mu \nu}$ are given by the following expressions:

$$
\begin{aligned}
{ }_{2} \mathcal{T}^{\mu \nu} & ={ }_{2} \mathcal{T}^{l l} l^{\mu} l^{\nu}+{ }_{2} \mathcal{T}^{m m} m^{\mu} m^{\nu}+{ }_{2} \mathcal{T}^{l m} l^{\mu} m^{\nu} \\
{ }_{-2} \mathcal{T}^{\mu \nu} & ={ }_{-2} \mathcal{T}^{n n} n^{\mu} n^{\nu}+{ }_{-2} \mathcal{T}^{m^{*} m^{*}} m^{* \mu} m^{* \nu}+{ }_{-2} \mathcal{T}^{n m^{*}} n^{\mu} m^{* \nu}
\end{aligned}
$$

with

$$
\begin{aligned}
& { }_{2} \mathcal{T}^{l l}=\frac{\rho_{\mathrm{in}}^{2}}{2}\left\{a^{2} \sin ^{2} \theta \frac{\partial^{2}}{\partial t_{\mathrm{in}}^{2}}-2 i a \sin \theta \frac{\partial^{2}}{\partial \theta \partial t_{\mathrm{in}}}+2 a \frac{\partial^{2}}{\partial \varphi_{\mathrm{in}} \partial t_{\mathrm{in}}}+\csc ^{2} \theta \frac{\partial^{2}}{\partial \varphi_{\mathrm{in}}^{2}}-\frac{\partial^{2}}{\partial \theta^{2}}-2 i \csc \theta \frac{\partial^{2}}{\partial \theta \partial \varphi_{\mathrm{in}}}\right. \\
& +\frac{2 i \cos \theta}{\sin ^{2} \theta} \frac{\partial}{\partial \varphi_{\mathrm{in}}}+\cot \theta \frac{\partial}{\partial \theta}+2 i a \sin \theta \frac{6 r-2 i a \cos \theta}{\Sigma}\left[i a \sin \theta \frac{\partial}{\partial t_{\mathrm{in}}}+\frac{\partial}{\partial \theta}+i \csc \theta \frac{\partial}{\partial \varphi_{\mathrm{in}}}\right. \\
& \text { - } \left.\left.\quad i a \sin \theta \rho_{\text {in }}^{*}\right]\right\} \text {, } \\
& { }_{2} \mathcal{T}^{m m}=-\left\{\left(\frac{\partial}{\partial t_{\text {in }}}-\frac{\partial}{\partial r}-\frac{6 r-10 i a \cos \theta}{\Sigma}\right)\left(\frac{\partial}{\partial t_{\text {in }}}-\frac{\partial}{\partial r}\right)+\frac{4}{\Sigma^{2}}\left(r^{2}-5 a^{2} \cos ^{2}-4 r i a \cos \theta\right)\right\}, \\
& { }_{2} \mathcal{T}^{l m}=\frac{-2 \rho_{\mathrm{in}}}{\sqrt{2}}\left\{\left(i a \sin \theta \frac{\partial}{\partial t_{\mathrm{in}}}+\frac{\partial}{\partial \theta}+i \csc \theta \frac{\partial}{\partial \varphi_{\mathrm{in}}}+\cot \theta+(2 r-3 i a \cos \theta) \frac{i a \sin \theta}{\Sigma}\right)\left(\frac{\partial}{\partial t_{\mathrm{in}}}-\frac{\partial}{\partial r}\right)\right. \\
& \left.-3 \rho_{\text {in }}\left(i a \sin \theta \frac{\partial}{\partial t_{\text {in }}}+\frac{\partial}{\partial \theta}+i \csc \theta \frac{\partial}{\partial \varphi_{\text {in }}}-\cot \theta\right)+\frac{i a \sin \theta \rho_{\text {in }}}{\Sigma}\left(6 r^{2}+5 i a r \cos \theta-5 a^{2} \cos ^{2} \theta\right)\right\}, \\
& { }_{-2} \mathcal{T}^{n n}=\frac{\rho_{\mathrm{in}}^{* 2}}{2}\left\{a^{2} \sin ^{2} \theta \frac{\partial^{2}}{\partial t_{\mathrm{in}}^{2}}+2 i a \sin \theta \frac{\partial^{2}}{\partial \theta \partial t_{\mathrm{in}}}+2 a \frac{\partial^{2}}{\partial \varphi_{\mathrm{in}} \partial t_{\mathrm{in}}}+\csc ^{2} \theta \frac{\partial^{2}}{\partial \varphi_{\mathrm{in}}^{2}}-\frac{\partial^{2}}{\partial \theta^{2}}+2 i \csc \theta \frac{\partial^{2}}{\partial \theta \partial \varphi_{\mathrm{in}}}\right. \\
& \left.-\cot \theta \frac{\partial}{\partial \theta}+2 i a \cos \theta \frac{\partial}{\partial t}+i a \rho_{\text {in }}^{*} \sin \theta\left[i a \sin \theta \frac{\partial}{\partial t_{\text {in }}}-\frac{\partial}{\partial \theta_{\text {in }}}+i \csc \theta \frac{\partial}{\partial \varphi_{\text {in }}}\right]-2 a^{2} \sin ^{2} \theta \rho_{\text {in }}^{2}\right\} \rho_{\text {in }}^{-4}, \\
& { }_{-2} \mathcal{T}^{m^{*} m^{*}}=-\frac{1}{4 \Sigma^{2}}\left\{\Delta_{+}\left(\Delta_{+} \frac{\partial}{\partial t_{\mathrm{in}}}+2 \Delta \frac{\partial}{\partial r}+4 a \frac{\partial}{\partial \varphi_{\mathrm{in}}}\right) \frac{\partial}{\partial t_{\mathrm{in}}}+\Delta\left(\Delta \frac{\partial}{\partial r}+4 a \frac{\partial}{\partial \varphi_{\mathrm{in}}}\right) \frac{\partial}{\partial r}+4 a^{2} \frac{\partial^{2}}{\partial \varphi_{\mathrm{in}}^{2}}\right. \\
& \left.+4 M\left(3 r^{2}+a^{2}\right) \frac{\partial}{\partial t_{\text {in }}}-2(r-3 i a \cos \theta) \frac{\Delta}{\Sigma}\left(\Delta_{+} \frac{\partial}{\partial t_{\text {in }}}+\Delta \frac{\partial}{\partial r}+2 a \frac{\partial}{\partial \varphi_{\mathrm{in}}}\right)-8 i a \cos \theta \rho_{\mathrm{in}} \frac{\Delta^{2}}{\Sigma}\right\} \rho_{\mathrm{in}}^{-4}, \\
& { }_{-2} \mathcal{T}^{n m^{*}}=\frac{\rho_{\text {in }}^{*}}{\sqrt{2} \Sigma}\left\{( \Delta _ { + } \frac { \partial } { \partial t _ { \text { in } } } + \Delta \frac { \partial } { \partial r } + 2 a \frac { \partial } { \partial \varphi _ { \text { in } } } + ( 2 r + 3 i a \operatorname { c o s } \theta ) \frac { \Delta } { \Sigma } - r + M ) \left(i a \sin \theta \frac{\partial}{\partial t_{\text {in }}}-\frac{\partial}{\partial \theta}\right.\right. \\
& \left.+i \csc \theta \frac{\partial}{\partial \varphi_{\mathrm{in}}}\right)+\left(\cot \theta-(3 r+i a \cos \theta) \frac{i a \sin \theta}{\Sigma}\right)\left(\Delta_{+} \frac{\partial}{\partial t_{\mathrm{in}}}+\Delta \frac{\partial}{\partial r}+2 a \frac{\partial}{\partial \varphi_{\mathrm{in}}}\right)+6 i a r \sin \theta \rho_{\mathrm{in}} \frac{\Delta}{\Sigma^{2}} \\
& \text { - } \left.2(r-M)\left(\cot \theta-(3 r+i a \cos \theta) \frac{i a \sin \theta}{\Sigma}\right)-(r+3 i a \cos \theta) \cot \theta \frac{\Delta}{\Sigma}\right\} \rho_{\text {in }}^{-4},
\end{aligned}
$$

where we have also act the ${ }_{-2} \mathcal{T}^{\mu \nu}$ operator on $\rho^{4}$.

Once obtained these perturbation equations for arbitrary sources within a description free of geometric divergencies, it is possible to proceed further and perform the numerical evolution of the $\Psi_{0}{ }^{(1)}$ and $\Psi_{4}{ }^{(1)}$ perturbed Weyl scalars for cases as interesting as the ones due to an orbiting object, or the one mention above of an inspiring and finally colliding and coalescing object, or those due to accreting matter, for mention some examples. These studies are currently under way.

We finish this presentation testing the perturbation equations Eqs. (19) in Schwarzschild described with the Kerr Schild coordinates, which implies setting the angular parameter $a=0$, and the Eqs.(19.21) reduce to the form:

$$
\begin{aligned}
& {\left[\Delta_{+} \frac{\partial^{2}}{\partial t_{\mathrm{in}}^{2}}-4 M r \frac{\partial^{2}}{\partial r \partial t_{\mathrm{in}}}-\Delta \frac{\partial^{2}}{\partial r^{2}}-\frac{\partial^{2}}{\partial \theta^{2}}-\frac{1}{\sin ^{2} \theta} \frac{\partial^{2}}{\partial \varphi_{\mathrm{in}}^{2}}-2\left((s+1)(r-M)+2 s \Delta \varepsilon_{\mathrm{in}}\right) \frac{\partial}{\partial r}\right.} \\
& \left.-2(s r+(s+1) M) \frac{\partial}{\partial t_{\mathrm{in}}}-\cot \theta \frac{\partial}{\partial \theta}-2 \operatorname{si} \frac{\cos \theta}{\sin ^{2} \theta} \frac{\partial}{\partial \varphi_{\mathrm{in}}}-s\left(1-s \cot ^{2} \theta\right)\right]_{s} \psi=16 \pi \Sigma_{s} \mathcal{T}^{\mu \nu} T_{\mu \nu}{ }^{(1)},
\end{aligned}
$$


with $\Delta_{+}=r^{2}+2 M r$, the angular coordinate $\varphi$ remains unchanged in this case, and now $\Delta=r^{2}-2 M r$. The operators ${ }_{s} \mathcal{T}^{\mu \nu}$ are given by Eq. (20) but now the projected operators reduce to:

$$
\begin{aligned}
{ }_{2} \mathcal{T}^{l l}= & \frac{1}{2 r^{2}}\left\{\csc ^{2} \theta \frac{\partial^{2}}{\partial \varphi_{\mathrm{in}}^{2}}-\frac{\partial^{2}}{\partial \theta^{2}}-2 i \csc \theta \frac{\partial^{2}}{\partial \theta \partial \varphi_{\mathrm{in}}}+\frac{2 i \cos \theta}{\sin ^{2} \theta} \frac{\partial}{\partial \varphi_{\mathrm{in}}}+\cot \theta \frac{\partial}{\partial \theta}\right\} \\
{ }_{2} \mathcal{T}^{m m}= & -\left\{\left(\frac{\partial}{\partial t_{\mathrm{in}}}-\frac{\partial}{\partial r}-\frac{6}{r}\right)\left(\frac{\partial}{\partial t_{\mathrm{in}}}-\frac{\partial}{\partial r}\right)-\frac{4}{r^{2}}\right\} \\
{ }_{2} \mathcal{T}^{l m}= & -\frac{2}{\sqrt{2} r}\left\{\left(\frac{\partial}{\partial t_{\mathrm{in}}}-\frac{\partial}{\partial r}-\frac{3}{r}\right)\left(\frac{\partial}{\partial \theta}+i \csc \theta \frac{\partial}{\partial \varphi_{\mathrm{in}}}+\cot \theta\right)\right\} \\
{ }_{-2} \mathcal{T}^{n n}= & \frac{1}{2 r^{2}}\left\{\csc ^{2} \theta \frac{\partial^{2}}{\partial \varphi_{\mathrm{in}}^{2}}-\frac{\partial^{2}}{\partial \theta^{2}}+2 i \csc \theta \frac{\partial^{2}}{\partial \theta \partial \varphi_{\mathrm{in}}}+\cot \theta \frac{\partial}{\partial \theta}\right\} r^{4}, \\
{ }_{2} \mathcal{T}^{m^{*} m^{*}}= & -\frac{1}{4 r^{4}}\left\{\Delta_{+}\left(\Delta_{+} \frac{\partial}{\partial t_{\mathrm{in}}}+2 \Delta \frac{\partial}{\partial r}\right) \frac{\partial}{\partial t_{\mathrm{in}}}+\Delta^{2} \frac{\partial^{2}}{\partial r^{2}}+12 M r^{2} \frac{\partial}{\partial t_{\mathrm{in}}}-2 \frac{\Delta}{r}\left(\Delta_{+} \frac{\partial}{\partial t_{\mathrm{in}}}+\Delta \frac{\partial}{\partial r}\right)\right\} r^{4}, \\
{ }_{-2} \mathcal{T}^{n m^{*}}= & \frac{1}{\sqrt{2} r^{3}}\left\{\left(\Delta_{+} \frac{\partial}{\partial t_{\mathrm{in}}}+\Delta \frac{\partial}{\partial r}+2 \frac{\Delta}{r}-r+M\right)\left(-\frac{\partial}{\partial \theta}+i \csc \theta \frac{\partial}{\partial \varphi_{\mathrm{in}}}\right)\right. \\
& \left.+\cot \theta\left(\Delta_{+} \frac{\partial}{\partial t_{\mathrm{in}}}+\Delta \frac{\partial}{\partial r}\right)-2(r-M) \cot \theta-\cot \theta \frac{\Delta}{r}\right\} r^{4} .
\end{aligned}
$$

We try the simple case when the perturbation is due to dust radially falling unto the black hole, for which the stress energy tensor is given by

$$
T^{\mu \nu}=d(r, t) u^{\mu} u^{\nu}
$$

with $d(r, t)$ the density and $u^{\mu}$ the four velocity, which for the radially falling case has only radial and temporal components:

$$
u^{\mu}=\left(u^{t}, u^{r}, 0,0\right) .
$$

Projecting the stress energy tensor, Eq.(24), unto the null tetrad which for the Schawrzschild case is given by: (from Eq.(15) with $a=0$ )

$$
\begin{aligned}
l_{\mu} & =(1,-1,0,0), \\
n_{\mu} & =\frac{1}{2 r^{2}}\left(\Delta,-\left(r^{2}+2 M r\right), 0,0\right), \\
m_{\mu} & =\frac{r}{\sqrt{2}}(0,0,1, i \sin \theta),
\end{aligned}
$$

and taking into account the components different from zero of the four velocity, Eq.(25), we obtain that the stress energy tensor projections unto the null tetrad are,

$$
\begin{aligned}
T_{l l} & =d^{2}(r, t)\left(u^{0}-u^{r}\right)^{2}, \\
T_{n n} & =\frac{d^{2}(r, t)}{4 r^{4}}\left(\Delta u^{0}-\Delta_{+} u^{r}\right)^{2}, \\
T_{l m} & =T_{m m}=T_{n m^{*}}=T_{m^{*} m^{*}}=0 .
\end{aligned}
$$

Notice that the non zero projections are functions of $r$ and $t$ only. Thus, substituting the projections in the rhs of Eqs.(22), there is only left the action of the operators on $T_{l l}$ and on $T_{n n}$. But those operators for the Schwarzschild case are purely angular operators, and as the projections are functions of $(t, r)$, the action is zero. In this way, we conclude that radially infalling dust into the Schwarzschild black hole does not produce perturbations on the $\Psi_{0}{ }^{(1)}$ and $\Psi_{4}^{(1)}$ perturbed Weyl scalars for both cases. 
This result is expected considering that the gravitational waves carry quadrupolar radiation and higher, and that the radially infalling dust into the Schwarzschild black hole will produce only monopolar perturbations, which will not be reflected in the $\Psi_{0}{ }^{(1)}$ and $\Psi_{4}{ }^{(1)}$ perturbed Weyl scalars.

It can be seen that the same happens with a radially infalling massive scalar field, with stress energy tensor given by

$$
T_{\mu \nu}=\phi_{\mu} \phi_{\nu}-\frac{1}{2} g_{\mu \nu}\left(\phi^{\alpha} \phi_{\alpha}-2 V(\phi)\right),
$$

with the scalar field a function of $(t, r)$ only, and where subindex and superindex imply derivative with respect to $x^{\mu}$. Again, the only non null projections needed in the perturbation equation are

$$
T_{l l}=\left(\dot{\phi}+\phi^{\prime}\right)^{2} \quad \text { and } \quad T_{n n}=\frac{1}{4 r^{4}}\left(\Delta_{+} \dot{\phi}-\Delta \phi^{\prime}\right)^{2},
$$

where dot and prime stand for derivatives with respect to $t$ and $r$ respectively. Once more, they are only functions of $(t, r)$, thus the action of the operators on them is zero, so the massive scalar field radially infalling unto the Schwarzschild black hole does not produce perturbations on the $\Psi_{0}{ }^{(1)}$ and $\Psi_{4}{ }^{(1)}$ perturbed Weyl scalars.

\subsection{Outgoing case}

In this section we present the analogous derivation of the field equations for the perturbed scalar projections $\Psi_{0}{ }^{(1)}$ and $\Psi_{4}{ }^{(1)}$ of the Weyl tensor, including sources. The procedure, although length, is straightforward and makes the corresponding steps as in the ingoing case. Thus, from the null vector naturally defined in the Kerr Schild form, Eq.(7), we construct the following contravariant null tetrad:

$$
\begin{aligned}
l_{\mu_{\text {out }}} & =\left(1,-1,0,-a \sin ^{2} \theta\right), \\
n_{\mu_{\text {out }}} & =\frac{1}{2 \Sigma}\left(\Delta,-\left(r^{2}+a^{2}\left(\cos ^{2} \theta-\sin ^{2} \theta\right)+2 M r\right), 0,-a \Delta \sin ^{2} \theta\right), \\
m_{\mu_{\text {out }}} & =\frac{1}{\sqrt{2}(r+i a \cos \theta)}\left[-i a \sin \theta, i a \sin \theta, \Sigma, i\left(r^{2}+a^{2}\right) \sin \theta\right] .
\end{aligned}
$$

Thus, the covariant null tetrad is:

$$
\begin{aligned}
l_{\text {out }}^{\mu} & =(1,1,0,0), \\
n_{\text {out }}^{\mu} & =\frac{1}{2 \Sigma}\left(r^{2}+a^{2}+2 M r,-\Delta, 0,2 a\right), \\
m_{\text {out }}^{\mu} & =-\frac{1}{\sqrt{2}(r+i a \cos \theta)}(i a \sin \theta, 0,1, i \csc \theta) .
\end{aligned}
$$

From which we obtain the corresponding differential operators:

$$
\begin{aligned}
\mathbf{D}_{\text {out }} & =l^{\mu}{ }_{\text {out }} \partial_{\mu}=\partial_{t_{\text {out }}}+\partial_{r}, \\
\boldsymbol{\Delta}_{\text {out }} & =n^{\mu}{ }_{\text {out }} \partial_{\mu}=\frac{1}{2 \Sigma}\left[\left(r^{2}+a^{2}+2 M r\right) \partial_{t_{\text {out }}}-\Delta \partial_{r}+2 a \partial_{\varphi_{\text {out }}}\right], \\
\delta_{\text {out }} & =m^{\mu}{ }_{\text {out }} \partial_{\mu}=\frac{\rho^{*}{ }_{\text {out }}}{\sqrt{2}}\left(i a \sin \theta \partial_{t_{\text {out }}}+\partial_{\theta}+i \csc \theta \partial_{\varphi_{\text {out }}}\right),
\end{aligned}
$$

with the spinor coefficient $\rho_{\text {out }}$ defined below in Eq.(33).

The spinor coefficients are computed directly from their definition [1, 2], obtaining:

$$
\begin{aligned}
& \kappa_{\text {out }}=\sigma_{\text {out }}=\lambda_{\text {out }}=\nu_{\text {out }}=\varepsilon_{\text {out }}=0, \\
& \rho_{\text {out }}=-\frac{1}{r-i a \cos \theta}, \quad \mu_{\text {out }}=\frac{\Delta}{2 \Sigma} \rho_{\text {out }}, \quad \gamma_{\text {out }}=\mu+\frac{r-M}{2 \Sigma}, \\
& \pi_{\text {out }}=-\frac{i a \sin \theta}{\sqrt{2}} \rho_{\text {out }}{ }^{2}, \quad \beta_{\text {out }}=\frac{\cot \theta}{2 \sqrt{2}} \rho_{\text {out }}{ }^{*}, \quad \alpha_{\text {out }}=\pi_{\text {out }}-\beta^{*}{ }_{\text {out }}, \quad \tau_{\text {out }}=\frac{i a \sin \theta}{\sqrt{2} \Sigma},
\end{aligned}
$$


and finally, the only non zero Weyl scalar is:

$$
\Psi_{2 \text { out }}=M \rho_{\text {out }}{ }^{3} .
$$

As mentioned above, the Teukolsky equation, that is the field equation the perturbed scalar projections $\Psi_{0}{ }^{(1)}$ and $\Psi_{4}{ }^{(1)}$ of the Weyl tensor, including sources, Eq.(13), as well as the expression for the operators action on the sources, Eq. (20), are valid also for the outgoing case. Thus, using the geometric quantities given by Eqs.(32, 33, 34), and their properties given in the appendix B, Eq.(39,41) after a quite lengthily algebraic manipulation, we obtain the following equation for $\Psi_{0}{ }^{(1)}$ and $\Psi_{4}{ }^{(1)}$ perturbed Weyl scalars due to arbitrary source terms in outgoing coordinates:

$$
\begin{aligned}
& {\left[(\Sigma+2 M r) \frac{\partial^{2}}{\partial t_{\text {out }}^{2}}+4 M r \frac{\partial^{2}}{\partial r \partial t_{\text {out }}}-\Delta \frac{\partial^{2}}{\partial r^{2}}+2 a \frac{\partial^{2}}{\partial r \partial \varphi_{\text {out }}}-\frac{\partial^{2}}{\partial \theta^{2}}-\frac{1}{\sin ^{2} \theta} \frac{\partial^{2}}{\partial \varphi_{\text {out }}^{2}}\right.} \\
& -2(s+1)(r-M) \frac{\partial}{\partial r}+2(s r+(s+1) M+s i a \cos \theta) \frac{\partial}{\partial t_{\text {out }}}-\cot \theta \frac{\partial}{\partial \theta} \\
& \left.-2(s+1) i \frac{\cos \theta}{\sin ^{2} \theta} \frac{\partial}{\partial \varphi_{\text {out }}}-s\left(1-s \cot ^{2} \theta\right)\right]_{s} \psi=16 \pi \Sigma_{s} \mathcal{T}^{\mu \nu} T_{\mu \nu}{ }^{(1)},
\end{aligned}
$$

where ${ }_{s} \mathcal{T}^{\mu \nu}$ are given again by Eq.(20), but using the outgoing terms, the projected operators have now the following expressions:

$$
\begin{aligned}
& { }_{2} \mathcal{T}^{l l}=-\frac{\rho^{* 2}{ }_{\text {out }}}{2}\left[i a \sin \theta\left(i a \sin \theta \frac{\partial}{\partial t_{\text {out }}}+2 \frac{\partial}{\partial \theta}+2 i \csc \theta \frac{\partial}{\partial \varphi_{\text {out }}}\right) \frac{\partial}{\partial t_{\text {out }}}+\sin \theta \frac{\partial}{\partial \theta}\left(\csc \theta \frac{\partial}{\partial \theta}\right)\right. \\
& +2 i \frac{\partial}{\partial \theta}\left(\csc \theta \frac{\partial}{\partial \varphi_{\text {out }}}\right)-\csc ^{2} \theta \frac{\partial^{2}}{\partial \varphi_{\text {out }}^{2}}+2 \frac{i a \sin \theta}{\Sigma}(3 r+i a \cos \theta)\left(i a \sin \theta \frac{\partial}{\partial t_{\text {out }}}\right. \\
& \left.\left.+\frac{\partial}{\partial \theta}+i \csc \theta \frac{\partial}{\partial \varphi_{\text {out }}}-i a \sin \theta \rho_{\text {out }}^{*}\right)\right] \\
& { }_{2} \mathcal{T}^{m m}=-\left(\frac{\partial}{\partial t_{\text {out }}}+\frac{\partial}{\partial r}+\frac{3 r+i a \cos \theta}{\Sigma}\right)\left(\frac{\partial}{\partial t_{\text {out }}}+\frac{\partial}{\partial r}\right)-\frac{4}{\Sigma}, \\
& { }_{2} \mathcal{T}^{l m}=-\frac{\rho^{*}{ }_{\text {out }}}{\sqrt{2}}\left[\left(\frac{\partial}{\partial t_{\text {out }}}+\frac{\partial}{\partial r}+\frac{3 r+i a \cos \theta}{\Sigma}\right)\left(i a \sin \theta \frac{\partial}{\partial t_{\text {out }}}+\frac{\partial}{\partial \theta}+i \csc \theta \frac{\partial}{\partial \varphi_{\text {out }}}-\cot \theta\right)\right. \\
& \left.-2 i a \sin \theta\left(2 \rho_{\text {out }} \frac{\partial}{\partial t_{\text {out }}}+\rho_{\text {out }}^{* 2}\right)\right] \text {, } \\
& { }_{-2} \mathcal{T}^{n n}=-\frac{\rho^{2}{ }_{\text {out }}}{2}\left[i a \sin \theta\left(i a \sin \theta \frac{\partial}{\partial t_{\text {out }}}-2 \frac{\partial}{\partial \theta}+2 i \csc \theta \frac{\partial}{\partial \varphi_{\text {out }}}\right) \frac{\partial}{\partial t_{\text {out }}}+\sin \theta \frac{\partial}{\partial \theta}\left(\csc \theta \frac{\partial}{\partial \theta}\right)\right. \\
& -\csc ^{2} \theta \frac{\partial^{2}}{\partial \varphi_{\text {out }}^{2}}-\frac{i a \sin \theta}{\Sigma}(7 r+11 i a \cos \theta)\left(i a \sin \theta \frac{\partial}{\partial t_{\text {out }}}-\frac{\partial}{\partial \theta}+i \csc \theta \frac{\partial}{\partial \varphi_{\text {out }}}\right) \\
& \left.-2 i \frac{\partial}{\partial \theta}\left(\csc \theta \frac{\partial}{\partial \varphi_{\text {out }}}\right)+2 \rho_{\text {out }} \frac{a^{2} \sin ^{2} \theta}{\Sigma}(r+9 i a \cos \theta)\right] \\
& { }_{-2} \mathcal{T}^{m^{*} m^{*}}=-\frac{1}{\Sigma^{2}}\left[\Delta_{+}\left(\Delta_{+} \frac{\partial}{\partial t_{\text {out }}}-2 \Delta \frac{\partial}{\partial r}+4 a \frac{\partial}{\partial \varphi_{\text {out }}}\right) \frac{\partial}{\partial t_{\text {out }}}+\Delta\left(\Delta \frac{\partial}{\partial r}-4 a \frac{\partial}{\partial \varphi_{\text {out }}}\right) \frac{\partial}{\partial r}+4 a^{2} \frac{\partial^{2}}{\partial \varphi_{\text {out }}^{2}}\right. \\
& +4 M\left(r^{2}-a^{2}\right) \frac{\partial}{\partial t_{\text {out }}}-2 \frac{\Delta}{\Sigma}(3 r+5 i a \cos \theta)\left(\Delta_{+} \frac{\partial}{\partial t_{\text {out }}}-\Delta \frac{\partial}{\partial r}+2 a \frac{\partial}{\partial \varphi_{\text {out }}}-2 i a \cos \theta \frac{\Delta}{\Sigma}\right) \\
& \left.+4 a(r-M) \frac{\partial}{\partial \varphi_{\text {out }}}+4 r(r+i a \cos \theta)\left(\frac{\Delta}{\Sigma}\right)^{2}\right] \\
& { }_{-2} \mathcal{T}^{n m^{*}}=-\frac{\rho^{*}{ }_{\text {out }}}{2 \sqrt{2} \Sigma}\left[\left(\Delta_{+} \frac{\partial}{\partial t_{\text {out }}}-\Delta \frac{\partial}{\partial r}+2 a \frac{\partial}{\partial \varphi_{\text {out }}}+2(r-M)-(5 r+3 i a \cos \theta) \frac{\Delta}{\Sigma}\right) \times\right.
\end{aligned}
$$




$$
\begin{aligned}
& \times\left(i a \sin \theta \frac{\partial}{\partial t_{\text {out }}}-\frac{\partial}{\partial \theta}+i \csc \theta \frac{\partial}{\partial \varphi_{\text {out }}}+\cot \theta-\left(3 r+5 i a \cos \theta \frac{i a \cos \theta}{\Sigma}\right)\right) \\
& \left.-3 i a \sin \theta\left(r^{2}+6 i a \cos \theta-a^{2} \cos ^{2} \theta\right) \frac{\Delta}{\Sigma^{2}}\right] .
\end{aligned}
$$

Again we recall that these equations were directly obtained working from the original field perturbation equation, Eqs.(19).

\section{Conclusions}

Scratching form the top, we have derived the equations for two of the radiative parts of the perturbed Weyl tensor, namely $\Psi_{0}{ }^{(1)}$ and $\Psi_{4}{ }^{(1)}$ in the Kerr spacetime described in the Kerr Schild coordinates, including sources. We have done son for the ingoing as well as for the outgoing descriptions.

Even thought the procedure followed in this work is straight forward, we consider that the equations obtained are a needed step in the research line of semi analytical studies of perturbed black holes, and will be useful for community.

The present description allows us to perform studies as near to the horizon as is wished, even inside the horizon for that matter, as long as the coordinates are regular. Also, there are several works which describe initial data, namely in the Kerr Schild description, so our work fits well with them. We remark the fact that the derived equations with sources presented in this work were obtained directly from the Bianchi identities within the general null tetrad formulation. The reason for this is that the spinors operators are not scalar quantities and the changes from one tetrad to another are not due to coordinate transformations only. Thus, it is not safe to use the equations for the perturbed components derived by S. Teukolsky, back in 1972 [3], where he used the Eddington Finkelstein coordinates (a description well suited for the asymptotic analysis, which is the one he performed), and use the coordinate transformation to describe those equation in the Kerr Schild coordinates.

Thus, we derived the equations and tested them in simple cases where we obtain the expected results, such as the fact that radial perturbations do not generate radiation. The development of realistic situations is currently under way. This work continues the program started by Campanelli et al. [6] where they studied the gravitational perturbation within a description free of coordinate singularities, without sources. We have introduce another null tetrad and derived the equations with arbitrary sources.

The analysis and the equations presented in this work are a complement for those performed purely numerically. Joint efforts are currently under way. As mentioned above, there are several phenomena that can now be studied from this perspective, mainly the most important is to continue in the study of gravitational waves from a compact star in circular, inspiral orbits around a massive spinning black hole, with applications to observation by LISA and LIGO [13], that is, to obtain predictions which could be detected and tested by the gravitational observatories which will start to work in the next year.

\section{Acknowledgments}

This work was partially supported by a CONACyT scholarship, and the grant DGAPA-UNAM IN121298. We want to thank Pablo Laguna for fruitful discussion during the elaboration of the present work.

\section{Appendix A}

The following commutation relations between the operators are repeatedly used in the work, and are valid for any algebraically special type $\mathrm{D}$ spacetime (we used that $\alpha=\pi-\beta^{*}$ ):

$$
\begin{gathered}
\mathbf{D} \boldsymbol{\Delta}=\boldsymbol{\Delta} \mathbf{D}-\left(\gamma+\gamma^{*}\right) \mathbf{D}-\left(\varepsilon+\varepsilon^{*}\right) \boldsymbol{\Delta}+\left(\tau+\pi^{*}\right) \delta+\left(\tau^{*}+\pi\right) \delta^{*}, \\
\delta \delta^{*}=\delta^{*} \delta+\left(\mu-\mu^{*}\right) \mathbf{D}+\left(\rho-\rho^{*}\right) \boldsymbol{\Delta}+\left(2 \beta-\pi^{*}\right) \delta-\left(2 \beta^{*}-\pi\right) \delta^{*},
\end{gathered}
$$




$$
\begin{gathered}
\left(\mathbf{D}+q \rho-\rho^{*}-(p+1) \varepsilon+\varepsilon^{*}\right)(\delta-p \beta+q \tau)-(\delta-p \beta+q \tau)(\mathbf{D}+q \rho-p \varepsilon)=0 \\
\left(\boldsymbol{\Delta}-q \mu+\mu^{*}+(p+1) \gamma-\gamma^{*}\right)\left(\delta^{*}-p \beta^{*}+(p-q) \pi\right) \\
-\left(\delta^{*}-p \beta^{*}+(p-q+1) \pi-\tau^{*}\right)(\boldsymbol{\Delta}-q \mu+p \gamma)=0
\end{gathered}
$$

where $p$ and $q$ are arbitrary real numbers. This expression are valid for the outgoing and ingoing case.

Finally, the following identities among the spinor coefficients are also useful:

$$
\begin{array}{ll}
\beta^{*} \tau=-\beta \pi, & \pi^{*} \rho=\tau \rho^{*}, \\
\mu^{*} \rho=\mu \rho^{*}, & 2 \rho \gamma-4 \beta \pi-2 \pi \tau-\Psi_{2}=0 .
\end{array}
$$

\section{Appendix B}

In the derivation of the field equations, is useful to determine the action of the operators on the different spinor coefficients. Its explicit form is obtained from teh expressions for the spinor coefficients given by Eq.(17, 33), and the operators given by Eq. (16, 32). Some of those actions are valid for both cases, the ingoing and the outgoing:

$$
\begin{array}{lll}
\mathbf{D} \rho^{*}=\rho^{* 2}, & \boldsymbol{\Delta} \tau^{*}=-\left(\mu+\mu^{*}\right) \tau^{*}, & \delta \rho=\tau \rho, \\
\delta^{*} \rho=-\pi \rho, & \delta \pi^{*}=2\left(\beta-\pi^{*}\right) \pi^{*}, & \delta^{*} \mu=-\left(2 \pi-\tau^{*}\right) \mu, \\
\delta \rho^{*}=\pi \rho, & \delta^{*} \pi=2\left(\beta^{*}-\pi\right) \pi, & \delta^{*} \tau^{*}=\left(2 \beta^{*}-\pi+\tau^{*}\right) \tau^{*} .
\end{array}
$$

The following expressions are valid the ingoing case:

$$
\begin{array}{lll}
\mathbf{D} \varepsilon=\frac{1}{2}\left(\rho^{2}-\rho^{-2}\right), & \mathbf{D} \beta=\frac{\rho}{2}\left(2 \beta+\tau-\tau^{*}\right), & \mathbf{D} \pi^{*}=\rho\left(\tau^{*}-\pi\right), \\
\boldsymbol{\Delta} \tau^{*}=-2 \mu^{*} \tau^{*}, & \boldsymbol{\Delta} \pi=\pi^{*}\left(\mu+\mu^{*}\right), & \boldsymbol{\Delta} \mu=-(\mu+2 \gamma) \mu, \\
\boldsymbol{\Delta} \mu^{*}=-\left(\mu^{*}+2 \gamma\right) \mu^{*}, & \boldsymbol{\Delta} \beta=-\frac{\mu^{*}}{2}\left(2 \beta^{*}+\tau-\tau^{*}\right), & \delta \mu=\mu\left(2 \tau-\pi^{*}\right), \\
\delta \varepsilon=\frac{\rho}{2}\left(\tau-\tau^{*}\right), & \delta^{*} \mu^{*}=\left(2 \tau^{*}-\pi\right) \mu^{*}, & \delta^{*} \Sigma^{-1}=2 \rho \tau^{*} \epsilon^{*} .
\end{array}
$$

And, for the outgoing case, the following expressions are valid

$$
\begin{array}{lll}
\mathbf{D} \beta=\beta \rho^{*}, & \mathbf{D} \pi^{*}=2 \pi^{*} \rho^{*}, & \boldsymbol{\Delta} \tau^{*}=-\left(\mu+\mu^{*}\right) \tau^{*}, \\
\boldsymbol{\Delta} \pi=-2 \pi \mu, & \boldsymbol{\Delta} \mu=-\left(\mu^{*}+2 \gamma\right) \mu, & \boldsymbol{\Delta} \mu^{*}=-(\mu+2 \gamma) \mu^{*}, \\
\boldsymbol{\Delta} \beta=-\mu^{*} \beta^{*}, & \delta \mu=\mu^{*}\left(2 \pi^{*}-\tau\right), & \delta^{*} \mu^{*}=-\left(2 \pi^{*}+\tau\right) \mu, \\
\delta \beta^{*}=-\mu \beta^{*} . & &
\end{array}
$$

\section{References}

[1] S. Chandrasekhar, The Mathematical Theory of Black Holes (Clarendon, Oxford, 1983).

[2] V. P. Frolov, Newman Penrose method in the General Relativity Theory, Science Academy USSR, 96, (1977) (in Russian).

[3] S. A. Teukolsky, Phys. Rev. Lett. 29, (1972) 1114. - Astroph. J., 185, 635 (1973).

[4] M. Alcubierre, B. Brügmann, T. Dramlitsch, J. A. Font, Ph. Papapdopoulos, E. Seidel, N. Stergioulas, and R. Takahashi, Phys.Rev. D 62 (2000) 044034, gr-qc/0003071. 
[5] P. Laguna, Phys.Rev. D 60 (1999) 084012. G. Khanna, J. Baker, R. Gleiser, P. Laguna, C. Nicasio,, H. P. Nollert, R. Price, J. Pullin, Phys.Rev.Lett. 83 (1999) 3581-3584.

[6] M. Campanelli, G. Khanna, P. Laguna, J. Pullin, and M. Ryan, Class.Quant.Grav., 18, (2001) $1543-1554, g r-q c / 0010034$.

[7] R. A. Matzner, M. F. Huq, and D. Shoemaker, Phys.Rev. D 59 (1998) 024015.

[8] C. W. Misner, K. S., Thorne, \& J. A. Wheeler, 1998, Gravitation, W.H. Freeman \& Co., 21 st. Printing.

[9] K. S. Thorne $g r-q c / 9506086$

[10] C. Moreno, Ph. D. Thesis.

[11] P. L. Chrzanowski, Phys.Rev. D 11, No. 8, (1975) 2042-2062.

[12] D. Núñez, Ph.D. thesis, Moscow State Univ. (1987).

[13] L. S. Finn, and K. S. Thorne, Phys.Rev. D, 62, (2000) 124021, gr-qc/0007074. 